\title{
Auto-eficácia para Abstinência e Tentação para Uso de Drogas Ilícitas: Uma Revisão Sistemática
}

\author{
Suzana Dias Freire ${ }^{1}$ \\ Margareth da Silva Oliveira² \\ Pontificia Universidade Católica do Rio Grande do Sul
}

\begin{abstract}
RESUMO - Estudos sobre o processo de mudança nos comportamentos aditivos abordam a auto-eficácia para abstinência e a tentação para o uso de substâncias como aspectos centrais ao entendimento das adições. Este estudo buscou a revisão sistemática de artigos que avaliaram a auto-eficácia (AE) para abstinência e a tentação para uso de drogas ilícitas. Nas bases de dados PubMed, PsychInfo e LILACS, foram utilizados termos: abstinence, self-efficacy, temptation e scale na busca de publicações que relacionassem os descritores a drogas ilícitas. Dos 13 artigos selecionados, cinco aplicaram o mesmo instrumento, três adaptaram medidas e os demais, diferentes escalas. Grande parte dessas ferramentas apresenta situações para serem avaliadas quanto à tentação para o uso ou auto-eficácia para abstinência de drogas em geral.
\end{abstract}

Plavras-chave: revisão de literatura; auto-eficácia; drogas ilícitas.

\section{Self-efficacy for Abstinence and Temptation to Use Illicit Drugs: A Systematic Review}

\begin{abstract}
Studies on the process of change in addictive behaviors address self-efficacy for abstinence and temptation to use drugs as central aspects. This study conducted a systematic review of scientific literature about abstinence self-efficacy (AE) and temptation to use illegal drugs. At the databases PubMed, PsychInfo and LILACS the terms abstinence, self-efficacy, temptation and scale were used in the search process for publications that related the descriptors to illicit drugs. Of the 13 selected articles, five used the same instrument, three adapted measures used to assess other behaviors, and the remaining five used different scales. Most of these tools presented risk situations to be answered according to the abstinence self-efficacy or temptation to use drugs in general, with no distinction among substances.
\end{abstract}

Keywords: literature review; self-efficacy; street drugs.

É crescente o desenvolvimento e a aplicação de instrumentos que avaliam os diversos aspectos da motivação para mudança de comportamento. Por ser multidimensional, a motivação não é um domínio único que possa ser medido por um só instrumento (Donovan \& Marlatt, 2005). O Modelo Transteórico (MTT) propõe a combinação de diferentes mecanismos em um referencial integrativo para o entendimento do processo dinâmico e complexo que envolve a intenção, a conquista e a manutenção das mudanças (Carbonari \& DiClemente, 2000; Prochaska \& DiClemente, 1982). Nos comportamentos aditivos, o modelo sistematiza a mudança intencional de comportamento pela combinação de fatores como: prontidão; estágios motivacionais; processos experienciais e comportamentais; tomada de decisão; auto-eficácia; aliança terapêtica; entre outros (DiClemente, Schlundt \& Gemmell, 2004; Oliveira, Calheiros \& Andretta, 2006; Prochaska, DiClemente \& Norcross, 1992; Sutton, 2001).

1 Psicóloga, Especialista em Psicoterapias Cognitivo-Comportamentais pela UNISINOS, Mestre em Psicologia Clínica pela Pontifícia Universidade Católica do Rio Grande do Sul.

2 Psicóloga Clínica, doutora em Ciências pela Universidade Federal de São Paulo-UNIFESP/EPM. Pós-Doutorado na University of Maryland - Baltimore County - UMBC. Professora adjunta do Programa de Pós-Graduação em Psicologia da Faculdade de Psicologia da Pontifícia Universidade Católica do Rio Grande do Sul. Endereço para correspondência: Avenida Ipiranga, 6681, prédio $11,9^{\circ}$ andar, sala 932 - CEP 90619-900 - Porto Alegre - RS - Brasil. Email: marga@pucrs.br
A recaída, como parte do processo de mudança, vem recebendo especial atenção através de duas abordagens principais. Uma classifica diferentes situações de risco para recaída e a outra avalia expectativas de eficácia nas situações de recaída (Velicer, DiClemente, Rossi \& Prochaska, 1990). A relevância da avaliação da auto-eficácia (AE) no entendimento das adições se destaca em diversas publicações (DiClemente, 1986; DiClemente et al., 2001; DiClemente, Fairhurst \& Piotrowski, 1995; Marlatt, Baer \& Quigley, 1995). Em 1977, com a publicação de Self-efficacy: towards a unifying theory of behavior change, Bandura complementa as teorias da época com um elemento que vem ganhando importância até a atualidade. A auto-eficácia foi conceituada ao incluir a idéia de que os indivíduos criam e desenvolvem percepções sobre si mesmo. Ditas percepções são utilizadas como instrumentos para os objetivos que se persegue e como meio de controle sobre o próprio ambiente. Desde então, ampliou-se a centralidade do papel que a cognição desempenha na capacidade das pessoas de construir a realidade, auto-regularem-se, codificar informações e executar comportamentos. AAE foi integrada à Teoria Social Cognitiva (Bandura, 1999), que é fundamentada na agência para o autodesenvolvimento, a adaptação e a mudança (Bandura, 2001; Bandura et al., 2008). Isso significa agenciar intencionalmente o próprio funcionamento e as circunstâncias de vida. Esta auto-regulação é composta de processos que atuam como determinantes recíprocos 
do comportamento e desempenham significativo papel na percepção e formação das próprias influências ambientais. $\mathrm{O}$ desenvolvimento e o exercício da agência pessoal fazem uso das experiências de domínio como principal veículo de mudança (Bandura, 1989).

As pessoas são motivadas e orientadas pela previsão de metas. Parte do seu funcionamento sofre influências da antecipação, ou seja, da expectativa de resultado. Esse fator pessoal ativamente interfere no meio de forma direta e indireta. A maneira como a pessoa interpreta os resultados de seu próprio comportamento altera seus ambientes e suas interpretações, que, por sua vez, modifica seus comportamentos futuros. Determinismo recíproco estabelece as inter-relações entre fatores pessoais (cognições, afetos e eventos biológicos), ambientais e comportamentais (Bandura et al., 2008). AE, entendida como parte dos fatores pessoais, é a expectativa no próprio desempenho para alcançar um resultado esperado (Bandura, 1989; Bandura, 1999). Em geral, são usados diferentes termos para referir o mesmo construto: $\mathrm{AE}$, confiança, autoconfiança, crenças de eficácia, expectativa de eficácia, percepção de eficácia ou simplesmente eficácia.

O papel da AE no abuso e dependência de substâncias psicoativas (SPA) é multifacetado. DiClemente et al. (1995) definem diferentes tipos de $\mathrm{AE}$ que merecem ser citados:

- AE para o enfrentamento (copping self-efficacy) refere-se à capacidade de lidar com situações específicas, como ser assertivo com amigos ou buscar ajuda quando estiver ansioso, ao invés de usar drogas;

- AE para o tratamento (treatment behavior self-efficacy) envolve a capacidade de desempenhar comportamentos relacionados ao tratamento, como controle de estímulos, registro de pensamentos, etc;

- AE para recuperação (recovery self-efficacy) é a habilidade da pessoa de recuperar-se de um lapso ou de uma recaída;

- AE para controle (control self-efficacy) refere-se à confiança em ser capaz de manter o comportamento aditivo sob controle, como beber moderadamente e resistir a um episódio de uso nocivo;

- AE para abstinência (abstinence self-efficacy) é a expectativa de ser capaz de abster-se do comportamento aditivo.

Em qualquer aplicação, é necessária a especificação de qual tipo de AE se está avaliando (Bogenschutz, Tonigan \& Miller, 2006). No presente estudo, apenas a AE para abstinência foi investigada.

Auto-eficácia é reconhecida, desde sua conceituação, como um mediador do desempenho em tarefas futuras. $\mathrm{Ou}$ seja, maior auto-eficácia resulta em melhor desempenho, e vice versa. Mudanças no nível de auto-eficácia podem predizer mudanças duradouras no comportamento, se houver habilidades e incentivos adequados (Velicer et al., 1990). Assim, a avaliação deste elemento como mecanismo de mudança tem sido explorada nos mais diversos comportamentos (Bogenschutz et al., 2006; Dolan, Martin \& Rohsenow, 2008; Demmel \& Rist, 2005; Murphy, Bentall, Ryley \& Ralley, 2003; Goldbeck, Myatt \& Aitchison, 1997; Demmel, Nicolai \& Jenko, 2006; Hodgins, Peden, \& Makarchuk, 2004).

Medidas de auto-eficácia variam para diferentes áreas de problemas, mas são tipicamente pontuadas como uma única soma dos escores indicados em cada situação (Velicer et al.,
1990). Annis e Graham (1988) destacam que a expectativa de eficácia está condicionada a contextos específicos, e por isso deve ser investigada em diferentes circunstâncias.

O presente estudo verificou a bibliografia científica dos últimos 20 anos para identificar publicações que acessaram a AE para abstinência e a tentação para o uso de drogas ilícitas.

\section{Método}

Foi conduzida uma busca de referências bibliográficas em três bases de dados computadorizadas: PubMed (MEDLINE), PsycInfo e LILACS, acessadas em maio de 2009, utilizando os termos: abstinence, self-efficacy, temptation e scale. Foram incluídos artigos originais publicados nos últimos 20 anos (entre janeiro de 1989 e maio de 2009), com resumos disponíveis em língua inglesa, portuguesa e espanhola, que relacionassem os descritores ao uso de substâncias psicoativas diferentes de álcool e tabaco.

Os estudos selecionados avaliavam a auto-eficácia referente à abstinência de drogas, o que levou à exclusão de auto-eficácia para: uso controlado, tratamento, prática de atividade física, controle de peso ou controle alimentar. Também não foram selecionados artigos envolvendo outras adições, como jogo patológico, comportamentos alimentares e sexuais. Igualmente foram excluídas questões outras que avaliavam auto-eficácia e tentação, como comportamentos dependentes, disruptivos, crenças religiosas, entre outros.

Os critérios de seleção incluíram artigos empíricos, publicados em periódicos científicos, nos quais os construtos de AE para abstinência e tentação foram aferidos em relação ao uso de drogas ilícitas. Estudos com álcool, tabaco e medicamentos, que não abordassem pelo menos uma substância ilícita não foram incluídos, bem como estudos teóricos, teses, e produções não disponibilizadas na língua inglesa, portuguesa ou espanhola.

Para esta revisão foram identificados os 13 artigos científicos que avaliaram empiricamente a AE para abstinência de drogas e a tentação para o uso de pelo menos uma droga ilícita. Revisando os textos de cada um dos artigos, os dados pertinentes foram extraídos e estão apresentados na Tabela 1.

\section{Resultados e Discussão}

A Tabela 2 apresenta o resultado da busca nas bases de dados.

Do total ilustrado na Tabela 2, foram excluídos estudos que não abordavam adições. Essa primeira triagem atentou para pesquisas que aferissem os construtos da $\mathrm{AE}$ e tentação relacionando ao uso de qualquer substância. Nos 107 estudos pré-selecionados, foi aplicado o critério relativo às drogas ilícitas. Assim, foram descartados os que se referiam especificamente à: tabaco (43 estudos); álcool (40 estudos); tabaco e álcool (3 estudos); e outras drogas como anfetaminas, opióides e derivados (6 estudos). Dois artigos não puderam ser localizados na língua inglesa, espanhola ou portuguesa. Restaram 13 pesquisas que atendiam à especificidade desta revisão: 10 referentes a álcool e drogas; 2, a maconha; e 1 sobre dependentes de cocaína. 
Grande parte dos estudos é americana, exceto um do Canadá e um do México, único disponibilizado em espanhol. Dos 13 encontrados, 10 artigos investigavam homens e mulheres, dois apenas homens, e a amostra de um deles era formada somente por mulheres, sendo o único que caracteriza uma etnia - índias americanas. Apenas uma pesquisa não utiliza amostra clínica e entrevistou adolescentes estudantes do ensino médio quanto à frequência de uso de álcool e maconha.

Não foram encontradas publicações entre os anos de 1989 a 1997; e entre 2000 a 2005, que atendessem aos critérios desta revisão. Entre 1997 e 2000, foram selecionados três artigos, sendo que a produção se concentrou a partir de 2005, conforme os dados apresentados na Tabela 1. Ainda que o construto da AE date de 1977 (Bandura, 1977), e sua aplicação na área das adições desde 1979 (Marlatt \& Gordon, 2009), grande parte dos estudos da época abordavam álcool e tabaco. A partir dos resultados positivos encontrados com estas substâncias, e do aumento da expressividade de outras drogas, fez-se necessária a ampliação do foco para as múltiplas adições.

As 13 produções selecionadas foram agrupadas por semelhanças de método e dos objetivos propostos, formando três categorias: (a) 4 estudos de seguimento, que buscaram o poder preditivo do construto de AE para abstinência; (b)
5 estudos de correlação, que investigaram associações entre AE para abstinência e/ou tentação para o uso com outras variáveis; (c) 4 estudos instrumentais, que apresentaram propriedades psicométricas de medidas que acessaram estes dois construtos.

\section{Estudos de Seguimento}

A capacidade preditiva da medida de AE no resultado de tratamentos é investigada em estudos prospectivos, de corte e ensaios clínicos com diversas populações. Foram triados, nesta revisão, os que buscaram a $\mathrm{AE}$ para abstinência de SPA ilícitas.

Chong e Lopez (2008) observaram os preditores de uso de drogas e álcool em 346 mulheres índias americanas após internação para tratamento da dependência. Utilizaram a AASE (Alcohol Abstinence Self-efficacy Scale - DiClemente, Carbonari, Montgomery \& Hughes, 1994) e DASE (Drug Abstinence Self-efficacy Scale - DiClemente et al., 1994) para acessar, respectivamente, AE para abstinência de álcool e drogas em geral, sem especificar a SPA. Ainda que na descrição dos instrumentos haja refência ao uso das escalas de tentação correspondentes, apenas os resultados

Tabela 1. Artigos sobre AE para abstinência e tentação para o uso de drogas ilícitas

\begin{tabular}{|c|c|c|c|c|c|}
\hline & Trabalho & Título & Amostra & Instrumentos & Principais resultados \\
\hline \multirow{4}{*}{ 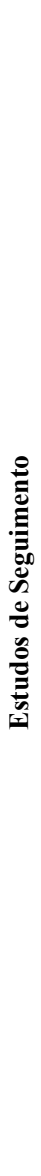 } & $\begin{array}{l}\text { Lozano, Ste- } \\
\text { phens e Roff- } \\
\text { man (2006) }\end{array}$ & $\begin{array}{l}\text { Abstinence and } \\
\text { moderate use goals } \\
\text { in the treatment of } \\
\text { marijuana depen- } \\
\text { dence. }\end{array}$ & $\begin{array}{l}-291 \text { adultos usuá- } \\
\text { rios de maconha em } \\
\text { tratamento de ambu- } \\
\text { latório } \\
77 \% \text { homens }\end{array}$ & $\begin{array}{l}\text {-TLFB (Time-Line Follow Back) } \\
\text {-MDS (Marijuana Dependence Scale) } \\
\text {-MPS (Marijuana Problem Scale) } \\
\text {-Algorítimo para definição de meta: abstinên- } \\
\text { cia X uso moderado } \\
\text {-Régua para avaliar AE para o resultado } \\
\text { escolhido }\end{array}$ & $\begin{array}{l}\text {-AE foi preditivo de resulta- } \\
\text { do em ambos os grupo: tanto } \\
\text { no grupo dos que escolheram } \\
\text { abstinência para meta do tra- } \\
\text { tamento como para o grupo } \\
\text { dos que escolheram a meta } \\
\text { do uso moderado. }\end{array}$ \\
\hline & $\begin{array}{l}\text { Jason, Davis e } \\
\text { Ferrari (2007) }\end{array}$ & $\begin{array}{l}\text { The need for sub- } \\
\text { stance abuse after- } \\
\text { care: Longitudinal } \\
\text { analysis of Oxford } \\
\text { House. }\end{array}$ & $\begin{array}{l}-170 \text { casas OH nas } 5 \\
\text { regiões dos EUA } \\
897 \text { residentes em } \\
\text { recuperação de álcool } \\
\text { e drogas } \\
67 \% \text { homens }\end{array}$ & $\begin{array}{l}\text {-TLFB (Time-Line Follow Back) adaptado } \\
\text { para avaliar residência, acesso a serviços de } \\
\text { saúde, além do uso de álcool e drogas. } \\
\text {-ASI (Addiction Severity Index) } \\
\text {-IPA (Important People and Activities Inven- } \\
\text { tory) } \\
\text {-AASE } \\
\text {-DASE }\end{array}$ & $\begin{array}{l}\text { Abstinência teve como } \\
\text { fatores preditivos: } \\
\text {-AE para abstinência } \\
\text {-rede de apoio adequada } \\
\text { para abstinência de álcool e } \\
\text { drogas } \\
\text {-duração do período de } \\
\text { residência na OH (permane- } \\
\text { cer na OH por pelo menos } 6 \\
\text { meses esteve relacionado a } \\
\text { maior AE e manutenção da } \\
\text { abstinência). }\end{array}$ \\
\hline & $\begin{array}{l}\text { Chong e Lopes } \\
(2008)\end{array}$ & $\begin{array}{l}\text { Predictors of re- } \\
\text { lapse for American } \\
\text { Indian women after } \\
\text { substance abuse } \\
\text { treatment. }\end{array}$ & $\begin{array}{l}\text {-346 mulheres } \\
\text { índias americanas em } \\
\text { tratamento de inter- } \\
\text { nação para DQ de } \\
\text { álcool e drogas. }\end{array}$ & $\begin{array}{l}\text {-ASI-NAV (Addiction Severty Índex Native } \\
\text { American Version) } \\
\text {-AASE / TUA } \\
\text {-DASE / TUD } \\
\text {-Itens selecionados do "Intake Questionnaire } \\
\text { of the Texas Christian University’s Criminal } \\
\text { Justice Program" }\end{array}$ & $\begin{array}{l}\text { Nos seguimentos de } 6 \text { e } 12 \\
\text { meses, altos escores de AE } \\
\text { para abstinência estavam } \\
\text { associados a menor proba- } \\
\text { bilidade de uso de álcool e } \\
\text { drogas. }\end{array}$ \\
\hline & $\begin{array}{l}\text { Litt, Kadden, } \\
\text { Kabela-Cor- } \\
\text { mier e Petry } \\
(2008)\end{array}$ & $\begin{array}{l}\text { Coping skills } \\
\text { training and contin- } \\
\text { gency management } \\
\text { treatments for mari- } \\
\text { juana dependence: } \\
\text { Exploring mecha- } \\
\text { nisms of behavior } \\
\text { change. }\end{array}$ & $\begin{array}{l}-240 \text { adultos com } \\
\text { diagnóstico de depen- } \\
\text { dência de maconha } \\
71 \% \text { homens }\end{array}$ & $\begin{array}{l}\text {-TLFB (Time-Line Follow Back) } \\
\text {-RTCQ (Readiness to Change Questionnaire) } \\
\text {-Adaptação de um questionário de AE ( } 20 \\
\text { itens, Likert } 7 \text { pontos) } \\
\text {-CSS (Coping Strategies Scale) }\end{array}$ & $\begin{array}{l}\text { Comparado com prontidão } \\
\text { para mudança e estratégias de } \\
\text { enfrentamento, o preditor de } \\
\text { abstinência mais significativo } \\
\text { ao longo dos períodos de } \\
\text { seguimento foi a AE medida } \\
\text { pré e pós- intervenção. }\end{array}$ \\
\hline
\end{tabular}


Tabela 1. Artigos sobre AE para abstinência e tentação para o uso de drogas ilícitas (continuação)

$\begin{array}{llll}\text { Alvarez, Alde- } & \text { Oxford House: Deaf- } & \text {-20 homens residentes de } & \text {-ASI (Addiction Severity Index) } \\ \text { banjo, Davi- } & \text { Affirmative Support } & \text { OH: } 10 \text { surdos e 10 não } & \text {-PSC (Perceived Sense of Com- } \\ \text { dson, Jason e } & \text { for Substance Abuse } & \text { surdos pareados por idade, } & \text { munity Scale) } \\ \text { Davis (2006) } & \text { Recovery. } & \begin{array}{l}\text { educação, etnia e tempo de } \\ \text { moradia na OH. }\end{array} & \text {-DASE } \\ & & \begin{array}{l}\text {-Em recuperação de álcool } \\ \text { e drogas }\end{array} & \end{array}$

Alvarez, Alde- $\quad$ Oxford House: Deaf- $\quad-20$ homens residentes de banjo, Davisurdos pareados por idade, educação, etnia e tempo de e drogas
A comparação dos grupos não mostrou diferenças em nenhuma das variáveis, exceto emprego.

Sem diferença significativa quanto a:

-anos de uso de álcool, cocaína e heroína -percepção de apoio social -AE para abstinência

\begin{tabular}{llc}
\hline $\begin{array}{l}\text { Naar-King, } \\
\text { Wright, Par- } \\
\text { sons, Frey, }\end{array}$ & $\begin{array}{l}\text { Transtheoretical } \\
\text { Model and substance } \\
\text { Templin e }\end{array}$ & $\begin{array}{l}\text {-64 pacientes de clínic } \\
\text { para tratamento do HIV }\end{array}$ \\
Ondersma & & -Entre 16 e 25 anos \\
(2006) & & $88 \%$ afro-americanos \\
& & $50 \%$ homens \\
& & $48 \%$ mulheres \\
& & \\
& &
\end{tabular}

-TLFB (Time-Line Follow Back)

-Estágios de motivação: algoritmo e régua (Rollnick's Readiness Ruler)

-AE para abstinência do álcool (3 itens) e drogas (3 itens) -Tentação pra usar álcool (20 itens) e drogas (20 itens)

-Social Provision Scale -Brief Symptom Inventory

-Tanto para álcool como para maconha, AE mediou a relação entre estágios de mudança e uso de SPA.

- AE, estágios de mudança e apoio social para evitar o álcool e maconha mostraram correlação significativa com uso de álcool e maconha e entre si no sentido esperado.

$\begin{array}{llll}\begin{array}{l}\text { Ouimette, } \\ \text { Coolhart, Fun- } \\ \text { derburk, Wade }\end{array} & \begin{array}{l}\text { Precipitants of first } \\ \text { substance use in }\end{array} & \begin{array}{c}-65 \text { pacientes internados } \\ \text { para tratamento da DQ. }\end{array} & \begin{array}{l}\text {-CAPS (Clinician Administered } \\ \text { PTSD Scale) } \\ \text { e Brown (2007) }\end{array} \\ \begin{array}{llll}\text { substance use disorder } \\ \text { patients with PTSD. }\end{array} & \begin{array}{l}\text { Com TEPT }(\mathrm{n}=32) \\ \text { Sem TEPT }(\mathrm{n}=33)\end{array} & \begin{array}{l}\text { da entrevista do Miller e Marlatt } \\ \text { (1996) para avaliar precipitantes } \\ \text { do uso. }\end{array} \\ & & 57 \% \text { mulheres } & \end{array}$

$43 \%(\mathrm{n}=66)$ álcool $37 \%(n=24)$ opióides $34 \%(\mathrm{n}=22)$ cocaína $15 \%(\mathrm{n}=10)$ sedativos $9 \%(\mathrm{n}=6)$ outras

Sujeitos com TEPT referiram circunstâncias diferentes associadas ao primeiro uso do que os pacientes sem TEPT: - enfrentamento de emoções negativas de natureza interpessoal

- uso para intoxicação

- expectativa de recaída - expectativas de AE menores: Sentiam menos confiança de resistir à vontade/impulso no futuro

Sentiam menos confiança em suas habilidades de lidar com situações particulares no futuro

Smyth e Wiechelt (2005)

-DIS (Diagnostic Interview

\section{Schedule)}

-36 homens e 14 mulheres da DQ de múltiplas drogas

$-62 \%(\mathrm{n}=31)$ com Transtorno de Personalidade $-38 \%(\mathrm{n}=19)$ sem Transtorno de Personalidade internados para tratamento

-DAST (Drug Abuse Screening Test)

-ADS (Alcohol Dependence

Scale)

-PDQ-R (Personality Disorder Questionnaire - Revised)

-IDTS (Inventory of Drug-Taking Situations)

-DTCQ (Drug-Taking Confidence Questionnaire)

-CISS (Coping Inventory for Stressful Situations)

-ICQ (Interpersonal Competence Questionnaire)

\begin{tabular}{|c|c|c|c|c|}
\hline $\begin{array}{l}\text { Aguilar e Pillon } \\
(2005)\end{array}$ & $\begin{array}{l}\text { Perception of temp- } \\
\text { tations to use drugs } \\
\text { among persons under } \\
\text { treatment. }\end{array}$ & $\begin{array}{l}\text {-61 pacientes de ambulató- } \\
\text { rio para tratamento da DQ. } \\
58 \text { homens } \\
3 \text { mulheres } \\
-50,8 \%(\mathrm{n}=31) \\
\text { Em abstinência } \\
-49,2 \%(\mathrm{n}=30) \\
\text { Estava usando SPA: } \\
\text {-álcool }(\mathrm{n}=11) \\
\text {-cocaína }(\mathrm{n}=6) \\
\text {-maconha }(\mathrm{n}=3) \\
\text {-tabaco }(\mathrm{n}=5) \\
\text {-outras }(\mathrm{n}=5)\end{array}$ & $\begin{array}{l}\text {-Entrevista para ver história de } \\
\text { uso de SPA } \\
\text {-Escala de Tentaciones de Uso de } \\
\text { Drogas }\end{array}$ & $\begin{array}{l}\text { Situações citadas como mais } \\
\text { tentadoras: } \\
\text {-ir a festas com os amigos } \\
\text {-estar em lugares onde todos } \\
\text { usam drogas } \\
\text {-estar com alguém que está } \\
\text { usando drogas } \\
\text {-sentir-se extremamente an- } \\
\text { sioso e estressado } \\
\text { Sentiram mais tentação: } \\
\text {-pacientes a menos tempo em } \\
\text { tratamento } \\
\text {-pacientes que usaram SPA } \\
\text { durante o tratamento }\end{array}$ \\
\hline
\end{tabular}

Quando comparados com o grupo sem Transtorno de Personalidade, os sujeitos com Transtorno de Personalidade tinham significativamente menos AE para manterem abstinência em situações envolvendo desconforto físico e conflitos interpessoais. 
Tabela 1. Artigos sobre AE para abstinência e tentação para o uso de drogas ilícitas (continuação)

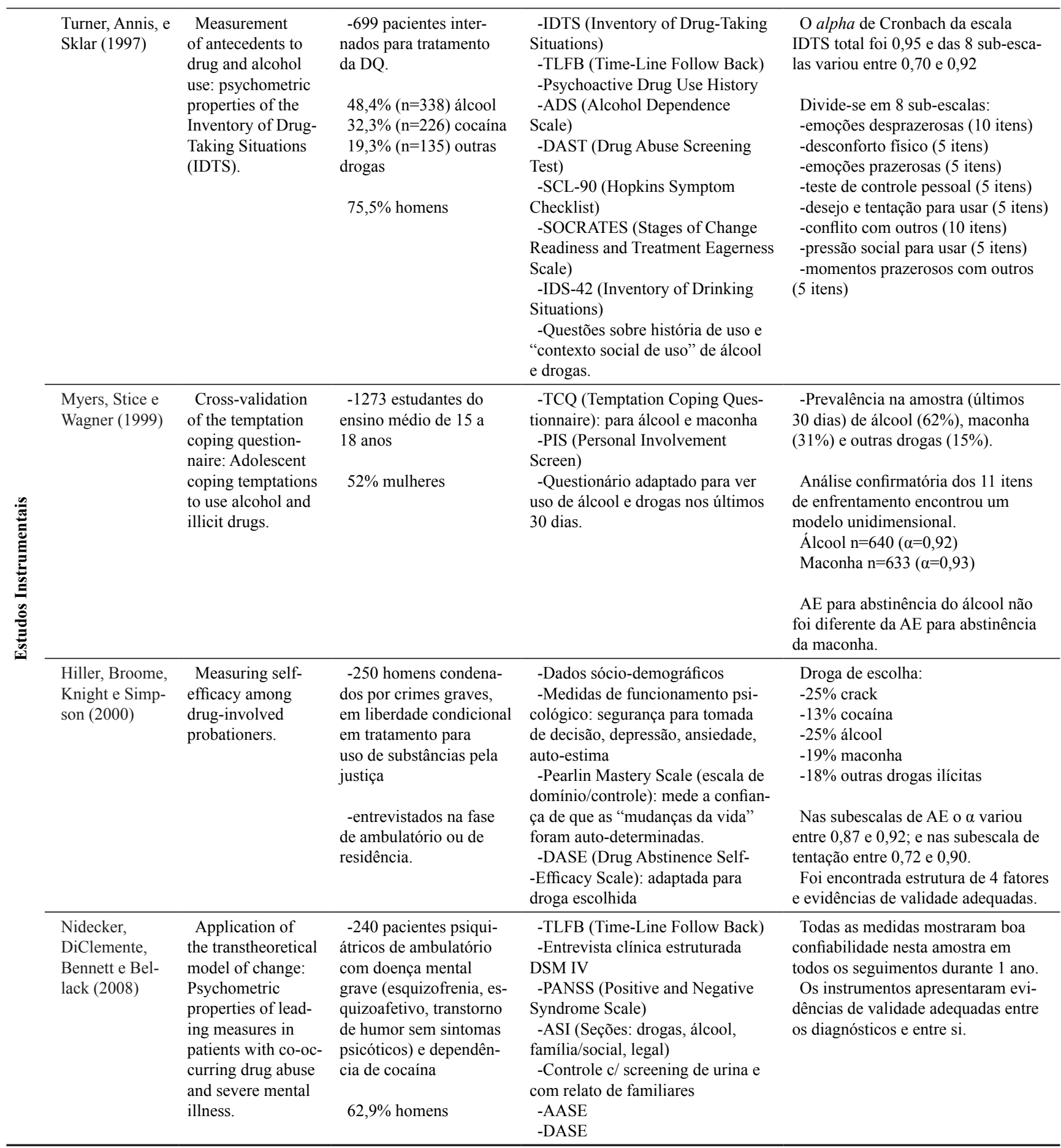

Tabela 2. Número de estudos encontrados em cada base de dados

\begin{tabular}{lccc}
\hline & PubMed $^{1}$ & PsychInfo $^{2}$ & Lilacs $^{3}$ \\
\hline Abstinence + Self-Efficacy + Scale & 21 & 94 & 2 \\
Temptation + Scale & 34 & 99 & 2 \\
Total & 55 & 193 & 4 \\
\hline
\end{tabular}

${ }^{1}$ PubMed: base de dados científicos da U.S. National Library of Medicine que incluí citações da MEDLINE.

${ }^{2}$ PsychInfo: base de consultas organizada pela American Psychological Association

${ }^{3}$ LILACS: Literatura Latino-Americana e do Caribe de Informação em Ciências da Saúde 
de AE foram apresentados, sendo omitidos os referentes à tentação. No seguimento de seis meses, altos escores de AE para abstinência estavam associados à menor probabilidade de uso de drogas $(\mathrm{OR}=0,53 ; \mathrm{p}=0,05)$. Da mesma forma, no follow up de 12 meses, altos escores de AE para abstinência estavam associados à menor probabilidade de uso de drogas $(\mathrm{OR}=0,49 ; \mathrm{p}<0,01)$. Aspecto interessante do estudo de Chong e Lopez (2008), com índias americanas, é a especificidade da amostra, que reconhece a necessidade de caracterizar o segmento cultural respeitando-se diferenças e particularidades. Quanto à forma de acessar a AE para abstinência, o uso da AASE e DASE possibilitou a distinção entre álcool e drogas. A aplicação da DASE para drogas em geral dificulta que se identifique se a substância para a qual foi respondido o instrumento está relacionada ou não à posterior abstinência. Assim, altos escores de AE para abstinência de crack podem não ser relacionados à posterior abstinência de maconha, por exemplo.

Outro estudo de seguimento de 12 meses foi conduzido em 170 casas Oxford House ${ }^{3}$, nas cinco regiões dos EUA, e entrevistou 897 residentes com dependência ou abuso de álcool e drogas. Os instrumentos usados também foram AASE e DASE. As escalas de tentação correspondentes não estão citadas e possivelmente não foram utilizadas. Também neste estudo, a DASE foi aplicada sem especificar a qual droga referia-se, o que implica na mesma limitação verificada no estudo de Chong e Lopez (2008). Com o uso de regressão logística, a AE para abstinência foi identificada como fator preditivo de abstinência tanto de álcool quanto de drogas. Constataram que a permanência de moradia na $\mathrm{OH}$, por seis meses pelo menos, esteve relacionada à maior $\mathrm{AE} \mathrm{e}$ manutenção da abstinência (Jason, Davis \& Ferrari, 2007).

Um ensaio clínico randomizado, com seguimento de 16 meses, avaliou 291 usuários de maconha em atendimento ambulatorial. Foram examinadas influências de duas variáveis nos resultados do tratamento: (a) metas pessoais para o tratamento (abstinência versus uso moderado); e (b) AE para atingir essa meta. A aferição da $\mathrm{AE}$ contou com régua de um único item, na qual foi graduada a confiança na capacidade de manter abstinência ou na capacidade de usar maconha moderadamente, dependendo do que havia sido previamente escolhido como meta. Ainda que pouco sensível, essa medida de AE para abstinência de maconha foi preditiva de resultado tanto no grupo dos que selecionaram como meta a abstinência, como para os que optaram pela meta do uso moderado (Lozano, Stephens \& Roffman, 2006).

Outro ensaio clínico randomizado com follow up de 14 meses acompanhou 240 adultos em tratamento para dependência de maconha. Duas abordagens foram testadas separadamente e em conjunto: TCC e Manejo de Contingências. Nessa pesquisa, a AE foi avaliada com a adaptação de um questionário de Stephens, Wertz e Roffman (1995) composto por 20 itens respondidos em escala Likert de sete pontos. Resultados positivos em ambas as modalidades de intervenção resultaram em mudanças na AE para a abstinência durante

3 Oxford House pode ser considerado um programa de recuperação de drogas em residências dirigidas e administradas por usuários de SPA em recuperação. Uso de nenhuma substância é tolerado, exceto tabaco. Então, os residentes de $\mathrm{OH}$ se mantêm em abstinência e moram lá durante o tempo que desejarem, desde que não usem álcool ou drogas. o tratamento. O preditor de abstinência mais significativo $(\mathrm{p}<0,001)$, ao longo dos períodos de seguimento, foi a $\mathrm{AE}$ medida pré e pós intervenção, quando comparado com outros fatores como prontidão para mudança e estratégias de enfrentamento. Com o objetivo de explorar o efeito desses fatores no sucesso dos tratamentos, a amostra foi restrita à dependência de maconha, aspecto que incrementa sua validade interna e reforça a necessidade de pesquisar cada SPA separadamente na investigação de mecanismos de mudança como a AE para abstinência (Litt, Kadden, Kabela-Cormier \& Petry, 2008).

Jason et al. (2007) aplicaram a AASE para álcool e a DASE para drogas em geral, e não fizeram uso das escalas de tentação correspondentes. Chong e Lopez (2008) utilizaram a DASE e a TUD também para drogas em geral, e não apresentaram os resultados de tentação, priorizando a investigação apenas da AE. Ambos os estudos buscaram o valor preditivo da AE para abstinência de drogas, sem evidenciar a SPA a que se referiam. É incontestável que essas escalas se comportam de maneira distinta de acordo com a droga para a qual são respondidas (DiClemente et al, 1995). Outros dois artigos (Lozano et al., 2006; Litt et al., 2008) mostraram formas diferentes de acessar o mesmo construto de AE para abstinência de maconha. Usaram instrumentos diferentes e apresentaram importantes características dos mecanismos de mudança no consumo de cannabis, que certamente difere do crack, do álcool, etc. A definição clara deste foco garante que a expectativa de eficácia observada referia-se à capacidade de abster-se de maconha, o que viabiliza especificamente o entendimento desta adição.

Apesar dos distintos delineamentos, é possível observar que, nas quatro produções agrupadas nesta categoria, em todas as amostras a AE foi preditiva de abstinência. Litt et al. (2008) concluem que tanto a AE quanto a abstinência devem ser focadas no tratamento, pois mudanças em uma leva a mudanças na outra.

\section{Estudos de Correlação}

Pesquisa com residentes de Oxford House, em recuperação de álcool e drogas, comparou 10 surdos e 10 não-surdos pareados por idade, educação, etnia e tempo de moradia na $\mathrm{OH}$. A comparação dos grupos não mostrou diferenças em nenhuma das variáveis, exceto quanto ao emprego $(\mathrm{p}<0,01)$. Nenhum dos participantes com surdez estava trabalhando, e oito dos não-surdos trabalhavam. Também não foi observada diferença quanto aos anos de uso de substâncias e percepção de apoio social. Foi usada a escala DASE e os residentes com e sem problemas auditivos não apresentaram diferenças quanto a AE para abstinência de drogas. Na descrição dos métodos, houve referência a uma modificação na AASE: foi trocada a palavra álcool por drogas, visando acessar ambas as substâncias. O instrumento foi aplicado uma única vez, para acessar indistintamente álcool e drogas. Visto o objetivo ser restrito à comparação entre surdos e não-surdos, a generalidade do instrumento não impacta em prejuízos significativos nos resultados. Diferente da categoria dos estudos anteriores, que buscavam o poder preditivo para a abstinência de determinada SPA, e por isso, se beneficiariam da especificidade 
dos instrumentos quanto à droga que investigavam. Ainda que a amostra tenha sido pequena e restrita ao sexo masculino, o estudo sugere que a $\mathrm{OH}$ oferece possibilidade de recuperação semelhante para pessoas com e sem problemas auditivos, quanto aos aspectos abordados nesta pesquisa (Alvarez, Adebanjo, Davidson, Jason \& Davis, 2006).

Um estudo com 36 homens e 14 mulheres, internados para o tratamento da dependência de drogas, comparou os sujeitos com diagnóstico de pelo menos um Transtorno de Personalidade $(62 \%, \mathrm{n}=31)$ com outros, sem esta comorbidade $(38 \%, \mathrm{n}=19)$. Pelo DTCQ (Drug-Taking Confidence Questionnaire) de Annis e Martin (1985), foi encontrado que os pacientes com Transtorno de Personalidade tinham menos AE para manterem abstinência em situações envolvendo desconforto físico $(\mathrm{p}<0,05)$ e conflitos interpessoais $(\mathrm{p}<0,05)$, quando comparados com sujeitos sem Transtorno de Personalidade. Como também apresentaram maior severidade de dependência de drogas $(\mathrm{p}<0,01)$, não é possível inferir se o risco aumentado para recaída nestas situações deve-se à presença da comorbidade ou à maior severidade da dependência de drogas. Os participantes foram instruídos a responder sobre a eficácia para abstinência da droga de preferência nas 50 situações descritas no instrumento. Ainda que as análises tenham sido conduzidas para drogas em geral, a instrução do instrumento restringia as respostas à droga de escolha, atentando para a importância de focar em uma SPA em avaliações de AE (Smyth \& Wiechelt, 2005).

No México, foi investigada a tentação para uso de drogas, em 16 situações ou circunstâncias específicas, com 61 pacientes ambulatoriais em tratamento para dependência de drogas. Destes, 49,2\% $(n=30)$ tinha consumido alguma SPA, dentre elas álcool $(n=11)$, cocaína $(n=6)$ ou outras drogas. Em escala Likert de cinco pontos, avaliaram a tentação percebida em cada situação (nada tentado - muito tentado). As situações que se destacaram como "muito tentadoras" foram: ir a festas com os amigos (21,3\%); estar em lugares onde todos usam drogas $(19,7 \%)$; estar com alguém que está usando drogas (18\%); e sentir-se extremamente ansioso e estressado (18\%). Foi observado que escores maiores de tentação estavam associados a estar a menos tempo em tratamento $(r=-0,32 ; \mathrm{p}=0,001)$. Pacientes que relataram ter usado SPA durante o tratamento apresentaram escores mais significativos $(t=3,26 ; p=0,002)$ quando comparados aos sujeitos que não usaram drogas no mesmo período. As medidas de tentação foram diferentes $(\mathrm{p}=0,004)$ de acordo com o tipo de droga que motivou a busca por tratamento. As diferenças de tentação de acordo com cada tipo de droga foram significativas $(\mathrm{p}<0,05)$ entre os que indicaram cocaína $(\mathrm{M}=20,19 ; \mathrm{DP}=19,34)$, maconha $(\mathrm{M}=42,04 ; \mathrm{DP}=29,21)$ e outras drogas $(\mathrm{M}=55,46 ; \mathrm{DP}=20,11)$. De acordo com a descrição do método, o instrumento sobre tentação para uso de drogas foi respondido de forma genérica, sem especificar a qual droga se referia. A SPA que motivou o tratamento foi identificada, porém sem estar condicionada ao questionário. Deste modo, os resultados referentes à tentação e à substância que motivou o tratamento merecem ser interpretados com restrições (Aguilar \& Pillon, 2005).

Outra pesquisa investigou pacientes comórbidos com diagnósticos de dependência química e Transtorno de Estresse Pós-Traumático (TEPT) e comparou com dependentes sem este duplo diagnóstico. Depois de terem alta de um tratamento de internação para uso de drogas, foram examinadas as razões, identificadas pelos próprios pacientes, para seu primeiro uso de SPA. Para tal foi conduzida uma análise qualitativa das razões para recaída, com a aplicação da taxonomia de Marlatt e Gordon (2009). Além disso, uma entrevista baseada na Relapse Interview (Miller \& Marlatt, 1996) investigou os precipitantes do uso de SPA nos 65 sujeitos que haviam recaído seis meses depois da alta. Destes, 32 haviam sido diagnosticados com TEPT e 33 não apresentavam esta comorbidade. Pacientes com TEPT relataram maior probabilidade de uso de SPA em resposta ao enfrentamento de emoções negativas de natureza interpessoal $(\mathrm{OR}=3,76$; $\mathrm{p}=0,05$; ) e menor probabilidade de uso $(\mathrm{p}=0,07)$ em situações que suscitam o desejo para o uso (cued urge). Tais pacientes relataram maior probabilidade de uso de SPA para intoxicação $(\mathrm{OR}=3,17 ; \mathrm{p}=0,06)$ e consideravam o primeiro uso como uma 'recaída' ( $\mathrm{OR}=4,67 ; \mathrm{p}=0,04)$ comparados aos pacientes sem TEPT. Pacientes com TEPT acreditavam que corriam mais risco de recair $(\mathrm{p}<0,05)$; sentiam menos confiança de resistir à vontade/impulso no futuro $(p<0,05)$; e sentiam menos confiança em suas habilidades de lidar com situações particulares no futuro $(\mathrm{p}<0,01)$ quando comparados com o outro grupo (Ouimette, Coolhart, Funderburk, Wade \& Brown, 2007).

Em ambulatório para tratamento do HIV, 64 jovens entre 16 e 25 anos foram entrevistados com o objetivo de investigar se construtos do MTT eram preditivos de uso de álcool e maconha nesta amostra. Nos 30 dias anteriores à avaliação, 38\% dos sujeitos haviam usado álcool e 30\% maconha. As frequências de uso de álcool e maconha estavam significativamente correlacionadas entre si $(p<0,01)$. Os estágios de motivação para mudança foram avaliados com uso de algoritmo e régua (Rollnick's Readiness Ruler). A AE para abstinência tanto de drogas quanto de álcool foram aferidas, cada uma, em 3 itens. Uma escala de tentação foi adaptada a partir de um questionário desenvolvido para investigar comportamentos de risco em adultos HIV positivos. Os mesmos 20 itens foram utilizados para avaliar a tentação para álcool e drogas. Descreviam situações de risco para uso e as respostas eram dadas em escala Likert de cinco pontos (nada tentado - extremamente tentado). A escala de tentação apresentou boa fidedignidade nessa amostra $(\alpha=0,92-0,96)$. Sobre o uso de maconha, as correlações significativas $(p<0,01)$ encontradas foram entre: $A E$ e régua de prontidão $(\mathrm{r}=0,70)$; AE e estágios de mudança $(\mathrm{r}=0,58)$; e AE e apoio para evitar drogas $(\mathrm{r}=0,48)$. Foram observadas correlações significativas $(\mathrm{p}<0,01)$ negativas entre: AE e tentação para usar maconha $(\mathrm{r}=-0,55)$; e frequência do uso nos últimos 30 dias $(\mathrm{r}=-0,60)$. A tentação para uso de maconha mostrou correlação significativa $(p<0,01)$ entre frequência do uso $(\mathrm{r}=0,78)$, régua de prontidão $(\mathrm{r}=-0,68)$ e estágios de mudança $(\mathrm{r}=-0,62)$. Conforme esperado a AE, os estágios de mudança e o apoio social para evitar álcool e maconha mostraram correlação significativa com a abstinência (Naar-King et al., 2006).

Dentre os cinco estudos de correlação, apenas o de Alvarez et al. (2006) fez uso da DASE para álcool e drogas em geral. Naar-King et al. (2006) referem outra forma de acessar AE: em três itens para uso do álcool e três para drogas, todos respondidos em escala Likert de cinco pontos que graduava 
a confiança para não usar SPA. No artigo de Smyth e Wiechelt (2005) este mesmo formato foi usado na medida da AE para abstinência em 50 situações. Ouimette et al. (2007) avaliaram AE em um único item, no qual a confiança para manter abstinência era graduada de zero a 100. Já a tentação foi avaliada, em dois estudos, de forma semelhante: os itens descrevem situações de risco e, em escala Likert de cinco pontos. Tanto no estudo de Aguilar e Pillon (2005) quanto no de Naar-King et al. (2006) as respostas graduavam a tentação para usar drogas. Independente da quantidade de itens, os estudos descrevem diferentes situações para serem respondidas quanto à tentação para usar ou quanto à confiança para não usar.

\section{Estudos Instrumentais}

O artigo de Nidecker, DiClemente, Bennett e Bellack (2008) demonstra a aplicabilidade de cinco instrumentos do MTT em dependentes de cocaína com comorbidades psiquiátricas graves. A AE para abstinência foi acessada com a escala reduzida de 12 itens DASE com excelente fidedignidade $(\boldsymbol{\alpha}=\mathbf{0 , 9 1})$. A Temptation to Use Drug Scale (TUDS) avaliou tentação para uso de cocaína, com a mesma fidedignidade $(\alpha=0,91)$ também na versão de 12 itens. Não foram apresentados valores para as subescalas, apenas para a escala total. Alguns fatores que contribuíram para os sólidos resultados deste estudo foram: a especificidade quanto ao uso de cocaína; a comparação entre dependentes e sujeitos em remissão da dependência de cocaína; e a confirmação de dados com familiares e com screening toxicológico. Sem igual rigor no controle de variáveis, Hiller, Broome, Knight e Simpson (2000) utilizaram as mesmas escalas na aferição de AE e tentação, em versão de 20 itens, para uso de drogas. $\mathrm{O}$ ponto forte deste estudo foi ter incluído questionamento para identificar a droga de escolha, sendo instruídos os sujeitos a responder as escalas de acordo com a substância por eles eleita. Os resultados apresentados não fizeram distinção por SPA, ainda que $38 \%$ da amostra tenha feito referência à cocaína (25\% fumada e $13 \%$ aspirada) como droga de escolha. Os alphas das subescalas variaram entre 0,87 e 0,92 (AE para abstinência) e entre 0,72 e 0,90 (tentação para uso) sugerindo boa fidedignidade sem explicitar à qual SPA se referem. Bzuneck (In Bandura et al., 2008) destaca a importância de aderir às condições específicas da realidade em estudo, ao construir/ adaptar escalas que avaliam AE, indicando que os itens devem detalhar de forma concreta o contexto a ser investigado. DiClemente et al. (1995) pontuam que a AE para abstinência sofre influência da substância e da situação a que se refere. O estudo canadense de Myers, Stice e Wagner (1999), atentaram a essa particularidade: AE para abstinência foi respondida em item para apenas uma situação de risco, com uma versão para álcool e outra para maconha. Mesmo que a medida de $\mathrm{AE}$ fosse parte de outra escala mais complexa, foram conduzidas análises específicas para correlacioná-la às demais variáveis. Ainda assim, na avaliação de uma única situação de risco, foi mantida a especificidade para o uso, separadamente, de álcool e maconha. Outro aspecto interessante desse estudo foi a distribuição, de forma randomizada, das versões de álcool e maconha entre os 1.273 estudantes. Com 640 adolescentes respondendo para álcool, e 633 para maconha, as médias de AE para abstinência destas duas substâncias não mostraram diferença significativa.

Do mesmo modo, a tentação para o uso de SPA está intimamente relacionada à droga a que se refere. Turner, Annis e Sklar (1997) apresentaram um inventário de situações de consumo (IDTS), com 50 circunstâncias a serem respondidas quanto à frequência de uso em cada ocasião. O diferencial é que todas as 50 situações foram respondidas para uma única SPA. Na versão computadorizada, é aplicado o mesmo instrumento para cada uma das três principais drogas escolhidas pelo entrevistado. A tentação para o uso forma uma das oito subescalas (Urges and Tentations to Use), avaliada em cinco itens respondidos em escala Likert de 4 pontos que gradua a frequência de uso.

Dos quatro estudos instrumentais, dois usaram as mesmas escalas (DASE e TUDS) e apresentaram resultados de forma distinta, o que dificultou a comparação entre eles (Nidecker et al., 2008; Hiller et al., 2000). De forma geral, ambos encontraram evidências de validade e fidedignidade para as escalas DASE e TUDS em amostras clínicas. Nos outros dois estudos instrumentais, os elementos de AE e tentação foram avaliados indiretamente, como parte integrante de ferramentas que avaliaram: (a) frequência do uso - a tentação formou uma subescala de cinco itens (Turner et al., 1997); e (b) enfrentamento da tentação - AE para abstinência foi avaliada em único item referente a apenas uma situação de risco (Myers et al., 1999).

Todas as produções agrupadas nesta categoria destacaram as substâncias para as quais as medidas estavam sendo investigadas. Nidecker et al. (2008) restringiram-se a dependentes de cocaína; Myers et al. (1999) randomizaram entrevistas para uso de álcool e maconha; Hiller et al. (2000) e Turner et al. (1997) incluíram questão para droga de escolha, condicionando as respostas à droga identificada pelo sujeito.

\section{Considerações Finais}

Na presente revisão, foram identificados os 13 artigos que apresentavam avaliações de AE para abstinência e tentação para o uso de SPA ilícitas. Apenas dois estudos não são americanos, sendo que grande parte das publicações ocorreu a partir de 2005 . Três categorias agruparam as produções por semelhanças quanto a objetivos e métodos: estudos de seguimento, de correlação e instrumentais. Cinco estudos, com pequenas variações, fizeram uso do mesmo instrumento. A escala AASE foi adaptada para o uso de drogas (DASE) e foi aplicada na versão de 12 e 20 itens. Outras medidas apresentaram formato semelhante ao descreverem situações de risco a serem respondidas quanto ao grau de eficácia para abstinência ou quanto ao grau de tentação para usar drogas em escala Likert de cinco pontos. Assim, dentre as produções aqui investigadas, esse foi o formato mais amplamente utilizado na aferição destes construtos: itens referentes a situações de risco graduados quanto a AE para abstinência ou tentação para o uso de substâncias.

Quanto à droga a que os instrumentos se referiam, cinco estudos não fizeram distinção entre as substâncias, o que evidencia pouca preocupação quanto ao detalhamento do 
contexto investigado (Jason et al., 2007; Chong \& Lopez, 2008; Alvarez et al., 2006; Ouimette et al., 2007; Aguilar \& Pillon, 2005). As análises conduzidas para drogas em geral devem ter seus resultados cuidadosamente interpretados. Dependendo dos objetivos do estudo, abordar SPA sem especificar qual delas, pode representar um fator de confusão importante. Tanto a AE quanto a tentação estão condicionadas à SPA a que se referem e a situações específicas, que devem ser bem descritas para o entendimento das particularidades de cada adição. Visto a relevância da aferição destes construtos, foram encontrados poucos artigos relacionando-os ao uso de substâncias ilegais. Conforme os resultados aqui reunidos, têm-se afirmado o importante papel desempenhado pelas avaliações desses elementos. $\mathrm{O}$ uso desses resultados configura uma área de aplicação e investigação promissora.

\section{Referências}

Aguilar, L. R., \& Pillon, S. C. (2005). Perception of temptations to use drugs among persons under treatment. Revista LatinoAmericana de Enfermagem, 13, 790-797.

Alvarez, J., Adebanjo, A. M., Davidson, M. K., Jason, L. A., \& Davis, M. I. (2006). Oxford House: Deaf-Affirmative Support for Substance Abuse Recovery. American Annals of the Deaf, 151(4), 418-422.

Annis, H. M., \& Graham, J. M. (1988). The Situational Confidence Questionnaire (SCQ-39) User's guide. Ontario, Canada: Addiction Research Foundation of Ontario.

Annis, H. M., \& Martin, G. (1985). Drug-Taking Confidence Questionnaire. Toronto, Canada: Addiction Research Foundation.

Bandura, A. (1977). Self-efficacy: towards a unifying theory of behavior change. Psychological Review, 84, 191-215.

Bandura, A. (1989). Human agency in social cognitive theory. American Psychologist, 44(9), 1175-1184.

Bandura, A. (1999). A sociocognitive analysis of substance abuse: an agentic perspective. Psychological Science, Special Section, 10(3), 214-217.

Bandura, A., Azzi, R. G., Polydoro, S. et al. (2008). Teoria social cognitiva: Conceitos básicos. Porto Alegre: Artmed.

Bogenschutz, M. P., Tonigan, J. S., \& Miller, W. R. (2006). Examining the effects of alcoholism typology and AA attendance on self-efficacy as a mechanism of change. Journal of Studies on Alcohol, 67(4), 562-567.

Bzuneck, J. A. (2008). Prefácio. In A. Bandura, R. G. Azzi, \& S. Polydoro et al. (Eds.) Teoria Social Cognitiva: Conceitos básicos (pp. 11-14). Porto Alegre: Artmed.

Carbonari, J. P., \& DiClemente, C. C. (2000). Using transtheoretical model profiles to differentiate levels of alcohol abstinence success. Journal of Consulting and Clinical Psychology, 68(5), 810-817.

Chong, J., \& Lopes, D. (2008). Predictors of relapse for American Indian women after substance abuse treatment. The Journal of the National Center, 14(3), 24-48.

Demmel, R., Nicolai, J., \& Jenko, D. M. (2006). Self-efficacy and alcohol relapse: concurrent validity of confidence measures, self-other discrepancies, and prediction of treatment outcome. Journal of Studies on Alcohol, 67(4), 637-641.
Demmel, R., \& Rist, F. (2005). Prediction of treatment outcome in a clinical sample of problem drinkers: Self-efficacy and coping style. Addictive Disorders \& Their Treatment, 4(1), 5-10.

DiClemente, C. C. (1986). Self-efficacy and the addictive behaviors. Journal of Social \& Clinical Psychology, 4(3), 302-315.

DiClemente, C. C., Carbonari, J. P., Daniels, J. W., Donovan, D. M., Bellino, L. E., \& Neavins, T. M. (2001). Self-efficacy as a matching hypothesis: Causal chain analysis. In R. Longabaugh, \& P. W. Wirtz (Eds.), Project MATCH Hypotheses: Results and Causal Chain Analyses (pp. 239-259). Project MATCH Monograph Series, 8. Rockville, MD: National Institute on Alcohol Abuse and Alcoholism.

DiClemente, C. C., Carbonari, J. P., Montgomery, R. P. G., \& Hughes, S. O. (1994). The Alcohol Abstinence Self-Efficacy Scale. Journal of Studies on Alcohol, 55(2), 141-148.

DiClemente, C. C., Fairhurst, S. K., \& Piotrowski, N. A. (1995). Self-efficacy and addictive behaviors. In J. E. Maddux (Ed.), Self-efficacy, adaptation and adjustment: theory, research and application (pp. 109-141). New York, NY: Plenum Press.

DiClemente, C. C., Schlundt, D., \& Gemmell, L. (2004). Readiness and stages of change in addiction treatment. The American Journal on Addictions, 13, 103-119.

Dolan, S. L., Martin, R. A., \& Rohsenow, D. J. (2008). Self-efficacy for cocaine abstinence: Pretreatment correlates and relationship to outcomes. Addictive Behaviors, 33, 675-688.

Donovan, D. M., \& Marlatt, G. A. (2005). Assessment of addictive behaviors. New York, NY: The Guilford Press.

Goldbeck, R., Myatt, P., \& Aitchison, T. (1997). End-of-treatment self-efficacy: A predictor abstinence. Addiction, 92(3), 313324.

Hiller, M. L., Broome, K. M., Knight, K., \& Simpson, D. D. (2000). Measuring self-efficacy among drug-involved probationers. Psychological Reports, 86, 529-538.

Hodgins, D. C., Peden, N., \& Makarchuk, K. (2004). Self-efficacy in pathological gambling treatment outcome: Development of a gambling abstinence self-efficacy scale (GASS). International Gambling Studies, 4, 99-108.

Jason, L. A., Davis, M. I., \& Ferrari, J. R. (2007). The need for substance abuse after-care: Longitudinal analysis of Oxford House. Addictive Behaviors, 32(4), 803-818.

Litt, M. D., Kadden, R. M., Kabela-Cormier, E., \& Petry, N. M. (2008). Coping skills training and contingency management treatments for marijuana dependence: Exploring mechanisms of behavior change. Addiction, 103(4), 638-648.

Lozano, B. E., Stephens, R. S., \& Roffman, R. A. (2006). Abstinence and moderate use goals in the treatment of marijuana dependence. Addiction, 101, 1589-1597.

Marlatt, G. A., \& Gordon, J. R. (2009). Prevenção de recaída: estratégias de manutenção no tratamento de comportamentos adictivos. Porto Alegre: Artmed.

Marlatt, G. A., Baer, J. S., \& Quigley, L. A. (1995). Self-efficacy and addictive behavior. In A. Bandura (Ed.), Self-Efficacy in Changing Societies (pp. 289-315). New York, NY: University Press.

Miller, W. R., \& Marlatt, G. A. (1996). Relapse interview - Intake and follow up. Addiction, 91, 231-240.

Murphy, P. N., Bentall, R. P., Ryley, L. D., \& Ralley, R. (2003). Predicting postdischarge opiate abstinence from admission measures of motivation and confidence. Psychology of Addictive Behaviors, 17(2), 167-170. 
Myers, M. G., Stice, E., \& Wagner, E. F. (1999). Cross-validation of the temptation coping questionnaire: Adolescent coping temptations to use alcohol and illicit drugs. Journal of Studies on Alcohol, 60(5), 712-718.

Naar-King, S., Wright, K., Parsons, J. T., Frey, M., Templin, T., \& Ondersma, S. (2006). Transtheoretical model and substance use in HIV-positive youth. AIDS Care 18(7), 839-845.

Nidecker, M., DiClemente, C. C., Bennett, M. E., \& Bellack, A. S. (2008). Application of the transtheoretical model of change: Psychometric properties of leading measures in patients with co-occurring drug abuse and severe mental illness. Addictive Behaviors, 33, 1021-1030.

Oliveira, M. S., Calheiros, P. R. V., \& Andretta, I. (2006). Motivação para mudança nos comportamentos aditivos. In B. S. G. Werlang, \& M. S. Oliveira (Eds.), Temas em Psicologia Clínica (pp. 115-124). São Paulo: Casa do Psicólogo.

Ouimette, P., Coolhart, D., Funderburk, J. S., Wade, M., \& Brown, P. J. (2007). Precipitants of first substance use in recently abstinent substance use disorder patients with PTSD. Addictive Behaviors, 32, 1719-1727.

Prochaska, J. O., \& DiClemente, C. C. (1982). Transtheorical therapy: Toward a more integrative model of change. Psychotherapy: Theory, Research and Practice, 20, 161-173.

Prochaska, J. O., DiClemente, C. C., \& Norcross, J. C. (1992). In search of how people change: Applications to addictive behaviors. American Psychologist, 47, 1102-1114.
Smyth, N. J., \& Wiechelt, S. A. (2005). Drug use, self-efficacy, and coping skills among people with concurrent substance abuse and personality disorders: Implications for relapse prevention. Journal of Social Work Practice in the Addictions, 5(4), 63-79.

Stephens, R. S., Wertz, J. S., \& Roffman, R. A. (1995) Self-efficacy and marijuana cessation: a construct validity analysis. Journal of Consulting and Clinical Psychology, 63, 1022-1031.

Sutton, S. (2001). Back to the drawing board? A review of applications of the transtheoretical model to substance use. Addiction, 96, 175-186.

Turner, N. E., Annis, H. M., \& Sklar, S. M. (1997). Measurement of antecedents to drug and alcohol use: Psychometric properties of the Inventory of Drug-Taking Situations (IDTS). Behavior Research Therapy, 35(5), 465-83.

Velicer, W. F., DiClemente, C. C., Rossi, J. S., \& Prochaska, J. O. (1990). Relapse situations and self-efficacy: An integrative model. Addictive Behaviour, 15, 271-283.

Recebido em 22.01.2010

Primeira decisão editorial em 11.06.2010

Versão final em 14.06.2010

Aceito em 26.06.2010 